\title{
De la longévité coopérative
}

Une étude de la survie des coopératives agricoles françaises

Cooperative longevity: A study of French agricultural cooperative survival

\author{
Myriam Carrère, Iragaël Joly et Damien Rousselière
}

Numéro 320, avril 2011

Le monde coopératif dans une économie plurielle : le problème de l'attractivité

Cooperatives in a plural Economy: The issue of their Attractiveness

URI : https://id.erudit.org/iderudit/1020910ar

DOI : https://doi.org/10.7202/1020910ar

Aller au sommaire du numéro

\section{Éditeur(s)}

Association Recma

ISSN

1626-1682 (imprimé)

2261-2599 (numérique)

\section{Découvrir la revue}

Citer cet article

Carrère, M., Joly, I. \& Rousselière, D. (2011). De la longévité coopérative : une étude de la survie des coopératives agricoles françaises. Revue internationale de l'économie sociale, (320), 82-98. https://doi.org/10.7202/1020910ar

\section{Résumé de l'article}

Le paysage coopératif agricole est en forte transformation depuis de nombreuses années, entre disparitions, fusions et consolidations. Différents travaux théoriques ont tenté de proposer une explication des particularités coopératives en termes d'efficacité et de capacité à survivre. Nous en présentons ici les grandes propositions de recherche. Notre travail est centré sur une contribution empirique. À ce titre, la mobilisation de méthodes simples d'analyse de survie permet de mettre en évidence une dynamique temporelle favorable aux coopératives vis-à-vis des autres entreprises avec lesquelles elles sont en concurrence. Cette dynamique s'accompagne de trajectoires sectorielles particulières et de dynamiques spécifiques suivant les régions. En conclusion, nous proposons quelques pistes d'explication de ces dynamiques. 


\title{
DE LA LONGÉVITÉ COOPÉRATIVE Une étude de la survie des coopératives agricoles françaises
}

\footnotetext{
* Ingénieure d'études, Institut national de la recherche agronomique, UMR Gael Inra. Mél.: myriam.carrere@grenoble. inra.fr.

* Maître de conférences, Grenoble, Institut national polytechnique, UMR Gael Inra. Mél.: Iragael.Joly@inpg.fr.

** Maître de conférences, Agrocampus Ouest, Institut national d'horticulture et de paysage, UMR Granem. Mél.: damien. rousseliere@agrocampus-ouest.fr.
}

\author{
par Myriam Carrère*, Iragaël Joly** et Damien Rousselière***
}

Le paysage coopératif agricole est en forte transformation depuis de nombreuses années, entre disparitions, fusions et consolidations. Différents travaux théoriques ont tenté de proposer une explication des particularités coopératives en termes d'efficacité et de capacité à survivre. Nous en présentons ici les grandes propositions de recherche. Notre travail est centré sur une contribution empirique. A ce titre, la mobilisation de méthodes simples d'analyse de survie permet de mettre en évidence une dynamique temporelle favorable aux coopératives vis-à-vis des autres entreprises avec lesquelles elles sont en concurrence. Cette dynamique s'accompagne de trajectoires sectorielles particulières et de dynamiques spécifiques suivant les régions. En conclusion, nous proposons quelques pistes d'explication de ces dynamiques.

a question de l'avenir du système coopératif agricole se pose plus que jamais: est-il une survivance du passé? Peut-il résister aux nouveaux modes de concurrence liés à la mondialisation (Côté, 2001 ; Draperi et Touzard, 2003; Koulytchizky et Mauget, 2003)? Ambiaud (2009) montre qu'en dépit des restructurations, dont la presse spécialisée se fait régulièrement l'écho, le profil général d'activité de la coopération agricole s'est peu modifié depuis dix ans, tout en prenant en compte certaines évolutions des industries agroalimentaires (comme la diversification des gammes de légumes) ou certains enjeux liés à l'environnement (comme la valorisation de la biomasse). Toutefois, de manière générale, deux résultats marquants interrogeant l'identité coopérative peuvent être mis en évidence. Le premier est celui concernant le contour du système coopératif avec la constitution de groupes coopératifs. Sur les dernières années, on a assisté à un vaste mouvement d'hybridation des statuts, de développement des unions de coopératives et de filialisation des activités par les coopératives auprès de sociétés commerciales (Filippi et al., 2008a). La complexification du périmètre coopératif conduit à ce que plus de la moitié des salariés se trouvent dans les filiales des coopératives agricoles. Le second résultat marquant concerne la disparition continue des petites coopératives sur les vingt dernières années (Ambiaud, 2007): près de la moitié des coopératives de moins de dix salariés ont en effet disparu. Cette dynamique est double, comme l'a montré Ambiaud (2007): une disparition pure et simple (cas des petites coopératives de commerce de gros) 
ou une disparition par fusion (cas des petites coopératives viticoles). Notre proposition a pour objet spécifique d'étudier la capacité à survivre de la forme coopérative dans un environnement concurrentiel mixte, c'est-à-dire caractérisé par une pluralité de formes d'organisation. Notre approche est donc une approche d'économie industrielle centrée sur la question de l'efficacité comparée des différents types d'entreprise (Maietta, Sena, 2008; Singh et al., 2001). Ce type d'approche s'oppose à celle de l'isomorphisme institutionnel postulant que les organisations évoluant dans un même environnement ont nécessairement tendance à se rapprocher (DiMaggio et Powell, 1983; Bager, 1994). Alors que de nombreux travaux théoriques ont porté sur les fondements théoriques de l'efficacité et de la capacité à survivre des coopératives en général (voir par exemple Hansmann, 1996) et des coopératives agricoles en particulier (voir par exemple Sykuta, Cook, 2001), peu de travaux empiriques ont cherché à tester sur la longue période les propositions théoriques observables que l'on pouvait tirer de cette littérature.

A cet effet, dans le cadre d'une mise à disposition par le ministère de l'Agriculture, nous nous appuyons sur les données confidentielles et quasi exhaustives de l'Enquête annuelle d'entreprise depuis 1984 et de l'enquête sur les petites coopératives agricoles depuis 1995. Notre méthodologie étant celle de l'économétrie des données de panel, nous utilisons plus particulièrement les modèles de survie (Allison, 1995; Hosmer, Lemeshow, 1999). Cette approche nous permet d'identifier les facteurs déterminant la survie des coopératives et de les distinguer ainsi des autres formes organisationnelles. Dans cet article, nous utilisons un modèle simple de survie (modèle non paramétrique de Kaplan-Meier [1958]) pour mettre en évidence des premiers faits stylisés concernant la dynamique des coopératives agricoles sur la période de 1984 à 2006.

Notre travail est dès lors développé en trois parties. A partir des explications données en économie industrielle à ces transformations du système coopératif, nous pouvons formuler deux premières conjectures de recherche. Ensuite, nous testons des modèles non paramétriques de survie différenciés (par statuts juridiques, par secteurs d'activité et par régions). En conclusion, nous discutons les différents résultats ainsi obtenus.

\section{L'état de la recherche sur les coopératives agricoles: quelle explication des transformations?}

La question de la survie des entreprises est une question de plus en plus développée de l'analyse économique (Moati et al., 1997). L'une des premières approches réside dans la croyance en une plus grande efficacité des coopératives dans la combinaison des différents facteurs de production: cette approche lie directement la survie à l'efficacité (une plus grande adaptation au contexte de production agricole). 
(1) Ce faisant, cette approche ne tient pas compte d'autres éléments comme la territorialisation de l'activité et la "nonopéabilisation " du capital de la coopérative, qui peuvent avoir de l'importance pour expliquer la survie des coopératives.

(2) Le pouvoir de marché ex ante (possibilité d'imposer un prix au-dessus du coût marginal), le pouvoir de marché ex postou lock in en raison d'investissements spécifiques réalisés en présence de contrats incomplets et l'asymétrie d'information, quand l'entreprise a une meilleure information que ses parties prenantes concernant la qualité de sa performance. (3) Le coût lié au contrôle de l'activité, les règles de prise de décision collective et l'aversion au risque. (4) L'oligopsone correspond à un marché où un grand nombre d'offreurs sont confrontés à un nombre réduit de demandeurs.

(5) La concentration des entreprises de débouchés (collecte et transformation des produits agricoles) pour les agriculteurs, accentuée par la saisonnalite de l'activité, confère un pouvoir important de négociation à ces entreprises au détriment des agriculteurs et explique notamment l'apparition des coopératives, mais aussi la transformation en coopératives d'autres entreprises (Hansmann, 1996, p. 134).

(6) La croissance des coopératives par croissance interne et croissance externe peut d'ailleurs conduire à remettre en cause ces deux avantages comparatifs de la forme coopérative vis-à-vis des autres formes de propriété.

(7) Une souplesse accrue du statut (comme l'incitation à la filiation développée par les lois de 19911992) est donc justifiée par cette approche.
Ainsi, l'approche s'inscrivant dans le courant de l'institutionnalisme du choix rationnel développée par Hansmann (1996) met l'accent sur certains facteurs d'efficacité dans la réduction de coûts liés à l'activité économique $^{(1)}$. L'existence d'une coopérative doit se comprendre dans le double choix (marché-entreprise et au sein des formes d'entreprise) qui se pose à tout acteur économique. Ces acteurs économiques potentiels sont dans le cas de l'agriculteur les différents acteurs de la chaîne de production (producteurs de matières premières, fournisseurs de produits phytosanitaires, distributeurs, transformateurs, consommateurs, mais aussi collectivités locales). Dès lors, pour Hansmann, la propriété d'une entreprise sera attribuée le plus efficacement à la classe de propriétaires de façon à minimiser la somme des coûts de propriété (2) pour cette classe et des coûts de marché( ${ }^{(3)}$ pour ceux qui ne sont pas propriétaires de l'entreprise. La raison de l'existence des coopératives agricoles tient alors à leur capacité à faire face à certains coûts de marché: elles sont un moyen de diminuer le pouvoir de marché du consommateur (oligopsone de la grande distribution ${ }^{(4)}$ ) en mettant en place une cartellisation entre les producteurs. En outre, les asymétries d'information sur certains produits agricoles sont relativement bien prises en compte par les coopératives de vente, qui peuvent inciter leurs membres producteurs à révéler les bonnes informations. Selon Hansmann, l'existence des coopératives agricoles tient d'abord aux très faibles coûts de propriété liés au pouvoir des agriculteurs ${ }^{(5)}$ : les coûts de monitoring seraient très faibles en raison de fortes incitations auprès des agriculteurs membres, les coûts de décision collective seraient également très faibles en raison de l'homogénéité forte de l'intérêt des parties prenantes ${ }^{(6)}$. Ces derniers éléments dus à la relation nouée avec leurs fournisseurs leur confèrent un avantage spécifique (notamment en cas de changement de la structure du marché), ce que Spear (2000) a développé avec la notion d'avantage coopératif. Le problème posé par l'horizon temporel dans les coopératives (en raison d'une aversion au risque qui peut conduire à un sous-investissement structurel) [voir par exemple Hansmann, 1999, ou Rey, Tirole, 2007] va toutefois conduire à ce que les coopératives soient désavantagées par rapport aux entreprises SA dès lors que ces stratégies impliquent des capitaux importants ${ }^{(7)}$ ou qu'elles prennent place en présence d'une hétérogénéité forte entre leurs membres. Se focalisant sur l'évaluation marchande de l'intérêt de la coopérative, cette approche néglige la possibilité pour les coopératives de mobiliser des ressources de différentes natures (marchandes et non marchandes). En outre, les objectifs qui leur sont assignés sont par nature différents de ceux des entreprises lucratives: maximisation d'un prix moyen des matières premières des adhérents, sécurisation des revenus face aux aléas des marchés, développement des capacités des sociétaires ou des groupes de sociétaires... Cette non-prise en considération de la diversité des logiques d'action peut être une approximation, certes dommageable, mais relativement courante, pour une entreprise à but lucratif. Elle est beaucoup moins acceptable pour les coopératives en particulier et l'économie 
(8) Même si la transformation de ces coopératives financières en banques universelles peut contribuer à distendre ce lien initialement fort (Vienney, 1994). sociale en général. A ce titre, l'institutionnalisme sociologique, courant comprenant notamment l'économie des conventions, souligne la particularité des compromis réalisés au sein d'une coopérative (Rousselière, Vézina, 2009; Rousselière, 2009), impliquant notamment une dimension civique ou domestique et une loyauté plus grande des membres dans la coopérative. Elle permet une évolution du système de rémunération au sein des coopératives agricoles, système légitime au regard des adhérents (Jarrigue, Touzard, 2001). Cette adaptabilité permise par la loyauté recherchée des parties prenantes peut expliquer des différentiels de survie dans des activités comme l'agriculture, qui ne peuvent être réduites à une simple activité économique, mais relèvent de composantes multidimensionnelles. Les coopératives disposent de plus d'un avantage stratégique dans leur capacité à nouer des compromis performants entre des objectifs pouvant être contradictoires (Boisard, Letablier, 1987).

Les propositions des approches évolutionnistes et régulationnistes complètent ce panorama rapide des approches institutionnalistes de la survie des coopératives en soulignant la complémentarité de ces organisations avec d'autres. Ainsi, les coopératives bénéficieraient plus que les autres d'un maillage local important d'institutions financières et notamment des coopératives de crédit agricole (Simons, Ingram, 2004; Gagliardi, 2009) ${ }^{(8)}$. Gagliardi (2009) aborde cette idée selon l'approche de la complémentarité institutionnelle (entre banques locales et coopératives), telle que développée par Boyer (2005). Dans ce cadre, André et Delorme (1983) ont montré, dans leur ouvrage sur l'évolution des interventions économiques de l'Etat en France au cours du $\mathrm{Xx}^{\mathrm{e}}$ siècle, le rôle essentiel dans la politique de l'agriculture joué par l'appui de l'Etat aux coopératives agricoles et au crédit agricole dans la modernisation des exploitations et des organisations collectives d'agriculteurs: "contribuer à maintenir la rentabilité des grandes exploitations et aider les moyennes exploitations à s'équiper et à améliorer leur compétitivité" (André, Delorme, 1983, p. 340).

Le fonds commun de ces approches relève finalement d'une première conjecture générale.

\section{Les coopératives ont une plus grande capacité à survivre que les autres types d'entreprise}

Cette proposition s'appuie donc sur ces différentes approches institutionnalistes donnant un avantage comparatif aux entreprises coopératives dans le secteur agroalimentaire et commercial. Elle nécessite d'être nuancée en raison de dynamiques territoriales ou régionales particulières. De manière parallèle, en effet, deux explications sont souvent données à la concentration des organisations coopératives, en liaison directe avec la manière dont les fusions sont considérées en économie industrielle (Mucchielli, Kohler, 2000): le pouvoir de marché et la recherche d'une baisse des coûts via les économies d'échelle ou les économies de gamme. Ces raisons affichées sont toutefois fortement controversées: 
(9) Les économies de champ sont de deux types. D'une part, elles sont constituées d'économies de variété, découlant de la production de biens différents, mais partageant des inputs communs. Elles impliquent généralement la mise en place d'équipements spécifiques, le développement de nouvelles compétences technologiques ou la capacité à pénétrer un nouveau marché D'autre part, elles sont constituées d'économies de gamme découlant de la production de plusieurs variétés d'un même produit générique différencié. Elles supposent d'atteindre un degré suffisant de flexibilité du processus de production et de réussir la politique commerciale d'accompagnement. Pour des variétés partageant de nombreuses caractéristiques techniques et commerciales, la maîtrise d'une catégorie donne I'essentiel des capacités pour en produire d'autres. si l'accroissement des parts de marché ou la baisse des coûts peuvent être des objectifs guidant les décideurs des coopératives, on peut toutefois se demander si le but recherché initialement (une réduction des coûts unitaires ou un gain de pouvoir de négociation) est réellement bien atteint (Briscoe, Ward, 2006).

La première explication est donc celle du pouvoir de marché. Le secteur agricole se caractérise en effet par l'existence d'un intermédiaire central entre les producteurs et les consommateurs: la grande distribution. Celle-ci exerce un pouvoir de marché par rapport aux producteurs et aux consommateurs. Réquillard (2007) a ainsi montré que les coopératives sont un moyen de réorganiser les producteurs et de lutter contre le pouvoir de marché de la grande distribution. Concernant notamment la production et le commerce de gros, cette question du pouvoir de marché dépend de la nature du produit (périssable ou semi-périssable) et de la taille du marché (volume des ventes). Ce renforcement de l'amont est même un des axes de la politique européenne dans le cadre de certaines organisations communes de marché relevant de la Politique agricole commune (PAC), et notamment dans le secteur des fruits et légumes (Bureau, 2007). Dans ce cadre, en diminuant ses achats de matières premières, la distribution peut jouer sur leur prix d'achat. De même, en diminuant ses ventes sur le marché final, elle pourra bénéficier d'un prix de vente supérieur (ou en pratiquant des prix plus élevés sur le marché final, elle vendra des quantités plus faibles). La quantité achetée par le distributeur se détermine alors au point d'intersection de la courbe de coût marginal d'achat (CMA), qui tient compte du fait qu'en réduisant ses achats le distributeur pourra bénéficier d'un prix plus faible (pouvoir de monopsone), et de la courbe de recette marginale du facteur (RMF), qui tient compte du fait qu'une réduction des ventes du distributeur ont un effet sur le prix de vente. Dans ce cadre, le prix payé au producteur est $\mathrm{p}_{\mathrm{la}}$ (au lieu de $\mathrm{p}_{0}$ dans une situation de concurrence parfaite) et les quantités achetées sont réduites $\left(\mathrm{q}_{1 \mathrm{a}}\right.$ au lieu de $\left.\mathrm{q}_{0}\right)$. Les consommateurs sont pénalisés, ainsi que les producteurs. Seuls les distributeurs profitent de cette situation et, finalement, le « bien-être » diminue par rapport à la situation de concurrence. La concentration est alors un moyen de faire face au pouvoir de la grande distribution (voir la figure 1, en page suivante).

Les économistes d'inspiration néoclassique voient toutefois cette proposition comme un "pis-aller " consécutif à l'incapacité d'introduction de la concurrence dans la grande distribution (voir par exemple Rey, Tirole, 2000). Ils mettent alors l'accent sur le potentiel d'une plus grande efficacité liée à une meilleure organisation de l'amont. Cette proposition est en lien avec la question des économies d'échelle et de champ caractéristiques de l'économie industrielle ${ }^{(9)}$.

Selon Mosheim (2008) et Rey et Tirole (2007), des coûts apparaissent dès lors que les coopératives n'ont pas la possibilité de discriminer les producteurs (libre entrée). Il leur est alors nécessaire de jouer sur les économies d'échelle par fusion des coopératives proches. En présence d'une liberté 


\section{Figure 1}

\section{L'impact de l'existence d'un pouvoir de marché par la grande distribution}

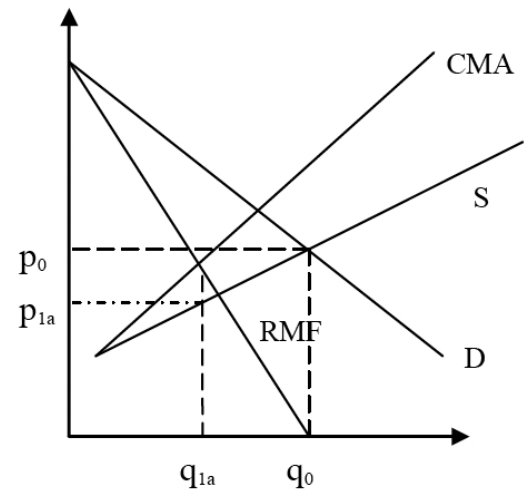

Source: Réquillard, 2007, p. 315.

d'acceptation de nouveaux adhérents, les coopératives peuvent également avoir intérêt à la fusion pour bénéficier soit d'économies d'échelle (par exemple pour des petites coopératives ayant des problèmes structurels de coûts) ou de champ (par exemple pour constituer des grands groupes polyvalents). C'est d'autant plus efficace que les coopératives ont initialement une exclusivité territoriale définie dans leurs statuts. Dans le cas du secteur viticole du Languedoc-Roussillon, Touzard (2000) a notamment mis en évidence que les fusions coopératives sont liées à la transformation de leur secteur d'activité. On voit ainsi que l'on peut expliquer la fusion de coopératives d'un même secteur d'activité pour la mutualisation de certains aspects (économie de gamme), ainsi que la fusion de coopératives de territoires voisins, contribuant à la création de grands groupes coopératifs à base territoriale (Harte, O'Connell, 2007).

Cette courte revue de la littérature nous autorise à émettre une autre proposition très générale.

\section{Cette capacité à survivre va varier selon le secteur et le territoire concerné}

Cette proposition précise la première dans le sens où l'avantage coopératif ne va pas être aussi important selon le secteur considéré. On peut notamment penser que dans les secteurs où une relation suivie avec les fournisseurs n'est pas un élément important, et donc là où la relation privilégiée des coopératives avec leurs adhérents fournisseurs de matières premières n'est plus un avantage décisif, les coopératives peuvent souffrir plus grandement de la concurrence. 
La seconde proposition rend bien compte de la capacité forte des coopératives à mobiliser certaines ressources du territoire. La tester suppose toutefois de bien faire attention à la manière dont la production agricole est spécialisée ou au contraire diversifiée selon les territoires. La comparaison des secteurs viticole (forte spécialisation territoriale) et laitier (plus grande diversification en raison de l'existence des quotas laitiers) est intéressante à ce propos.

\section{L'analyse empirique: une approche non-paramétrique de la survie des coopératives agricoles}

(10) Elle est à ce titre complétée par une enquête quinquennale sur l'ensemble des petites coopératives agricoles.

\section{Présentation des données}

Enquête riche sur l'activité économique et financière, l'Enquête annuelle d'entreprise permet l'accès à des données longitudinales sur les entreprises. Son intérêt est d'être obligatoire et exhaustive, ce qui à la différence d'une enquête non obligatoire ne nous expose pas à la présence de non-réponses non aléatoires et plus généralement d'erreurs liées à l'échantillonnage. Elle ne concerne toutefois que les entreprises de vingt salariés et plus et les coopératives de plus de dix salariés ${ }^{(10)}$. Elle est utilisée par le ministère de l'Agriculture dans la production de chiffres sur les coopératives agricoles (Ambiaud, 2007, 2009). Cette enquête a notamment fait l'objet d'une exploitation par Filippi et al. (2006, 2008a, 2008b) sur la constitution des groupes coopératifs et la logique de leur ancrage territorial.

Les observations dont nous disposons nous renseignent sur la durée pendant laquelle une organisation de production ou de commerce reste sous une forme juridique ou une autre. L'enquête recense, pour toutes les entreprises, les changements de statut ou les disparitions survenues entre 1984 et 2006. Etant donné cette restriction, la population enquêtée ne comprend que les entreprises encore en vie en 1984 et toutes celles créées depuis; certains événements ne sont donc pas observés, car en dehors de la période (création d'entreprise, disparition d'entreprise, changement de statut). Nous pouvons distinguer plusieurs cas, selon que nous avons une connaissance complète ou partielle des événements définissant la phase de présence de chaque entreprise dans l'échantillon. Tout d'abord, la présence sera complètement définie pour les entreprises dont nous observons la création ou le changement de forme juridique, ainsi que la fin de l'exercice ou un nouveau changement de forme juridique. Dans ce cas, aucune censure des durées n'est présente. Bien sûr, certaines organisations ne changent pas de forme juridique et ne disparaissent pas de l'échantillon. Elles sont présentes dans l'échantillon de la première date à la dernière. Ou encore, pour d'autres, seule la date de début de cycle est connue, avec la date d'interruption inconnue, ou l'inverse. Dans ces trois derniers cas, les durées sont donc censurées à droite (Kalbfleisch, Prentice, 1980), c'est-à-dire que les durées de survie sont supérieures ou égales à la durée d'observation. Ainsi, $17 \%$ des coopératives dans l'enquête sont nées 
(11) Une mesure plus rigoureuse de ce poids coopératif devrait également faire appel à la notion de part de marché ou de taille d'entreprise.
(12) De nombreuses possibilités sont offertes: stabilité de la mortalité (même risque de disparaître quel que soit l'âge), évolution monotone linéaire (augmentation ou diminution linéaire de la probabilité de disparaître), évolution accélérée ou décélérée, évolution non monotone (alternance de cycles de croissance et réduction de la probabilité de disparition).. (13) L'analyse multivariée non paramétrique n'existe que sous peu de logiciels à ce jour. Des logiciels comme SAS ou Stata ne proposent pas ce type d'analyse. avant 1984 et existent encore en 2006. Ce cas de figure est beaucoup plus rare pour les entreprises non coopératives (2,2\%). L'enquête renseigne la création de 49,9\% des coopératives et de 74,9\% des entreprises non coopératives.

Pour cette analyse, nous avons retenu trois conventions principales. Tout d'abord, nous avons codé en quatre grands secteurs d'activité les secteurs dans lesquels se retrouvent les coopératives. Il s'agit du commerce, du lait, de la viande et de la boisson. En cohérence avec la dimension exploratoire de notre étude, le caractère exemplaire des dynamiques au sein de ces secteurs nous a conduits à les retenir. Le secteur résiduel sera par définition beaucoup plus hétérogène et délicat à interpréter. Les entreprises étant classées dans un secteur d'activité suivant leur activité économique principale, nous ne prenons donc pas en compte le développement des structures polyvalentes. Ensuite, les statuts juridiques d'entreprise ont été regroupés en quatre grandes catégories: les coopératives, les SA non coopératives, les SARL non coopératives et les autres groupements (personnes physiques, autres personnes morales et personnes morales de droit public). Cette typologie s'appuie sur le type de contrôle de l'entreprise et la distribution des droits de propriété. Enfin, pour mettre en évidence les dynamiques territoriales, nous avons retenu le découpage géographique des fédérations régionales de coopératives agricoles. Ce découpage, s'inscrivant dans une histoire particulière de la coopération agricole, n'est utilisé que pour une analyse spécifique aux organisations coopératives.

Les premières statistiques descriptives montrent que la présence des coopératives dépend fortement du secteur. S'agissant d'un comptage du nombre d'entreprises (existant et ayant existé) sur la période étudiée (1984-2006), celle-ci recoupe seulement en partie la réalité du poids coopératif actuel dans chacun des secteurs ${ }^{(11)}$ (voir le tableau 1, en page suivante). Au total, nos données portant sur les industries agroalimentaires et celles du commerce conduisent à souligner la part importante des sociétés commerciales de type SA (52\% de l'ensemble des entreprises de la période 1984-2006) et SARL (32\%) dans l'ensemble des entreprises de plus de dix salariés (voir le tableau 2, en page suivante).

\section{Méthodologie}

L'analyse des durées de survie des coopératives agricoles et des entreprises non coopératives de notre échantillon se fonde sur la méthode dite d'analyse de survie ou encore des modèles de durée (Kalbfleisch, Prentice, 1980; Lawless, 2003; Hosmer, Lemeshow, 1999; Allison, 1995). Comme il s'agit de la première analyse de ce type, nous utiliserons une analyse non paramétrique ne formulant aucune hypothèse a priori sur la forme de la survie ${ }^{(12)}$. S'assimilant à ce que Heckman (2008) nomme une approche statistique (se différenciant d'une approche économétrique), cette analyse est toutefois limitée par nature dès lors que l'on introduit des facteurs explicatifs ${ }^{(13)}$. L'impact sur la survie de variables explicatives ne peut être quantifié. Seules les distributions des sous-populations peuvent être 
Tableau 1

Les entreprises par grands secteurs d'activité (cumulé 1984-2006)

\begin{tabular}{|c|c|c|c|c|c|c|c|}
\hline & \multicolumn{5}{|c|}{ Grands secteurs } & \multirow{2}{*}{ Total } \\
\hline & & Autre & Boisson & Commerce & Lait & Viande & \\
\hline \multirow{2}{*}{ Non coopératives } & $\mathrm{Nb}$ & 5167 & 889 & 299 & 1177 & 3888 & 11420 \\
\hline & $\%$ & 39,49 & 6,80 & 2,29 & 9,00 & 29,72 & 87,29 \\
\hline \multirow{2}{*}{ Coopératives } & $\mathrm{Nb}$ & 130 & 341 & 1000 & 143 & 49 & 1663 \\
\hline & $\%$ & 0,99 & 2,61 & 7,64 & 1,09 & 0,37 & 12,71 \\
\hline \multirow{2}{*}{ Total } & $\mathrm{Nb}$ & 5297 & 1230 & 1299 & 1320 & 3937 & 13083 \\
\hline & $\%$ & 40,49 & 9,40 & 9,93 & 10,09 & 30,09 & 100 \\
\hline
\end{tabular}

\section{Tableau 2}

\section{Les statuts juridiques}

\begin{tabular}{|l|r|r|}
\cline { 2 - 3 } \multicolumn{1}{c|}{} & $\mathbf{N b}$ & $\mathbf{\%}$ \\
\hline Autre & 434 & $3,3 \%$ \\
\hline Coopérative & 1663 & $12,7 \%$ \\
\hline SARL non coopérative & 4176 & $31,9 \%$ \\
\hline SA non coopérative & 6810 & $52,1 \%$ \\
\hline Total & 13083 & $100 \%$ \\
\hline
\end{tabular}

(14) La définition des marches de la fonction est donnée par le choix arbitraire des intervalles des temps d'interruption (ici les données sont collectées annuellement). Pour notre analyse, nous avons suffisamment de firmes à risque et de firmes subissant une interruption chaque année pour définir des classes d'un an.

(15) La prise en compte des observations censurées à droite est réalisée de telle sorte qu'elles contribuent à la population à risque jusqu'à ce qu'elles en disparaissent, mais ne sont pas comptabilisées comme des interruptions. II en résulte qu'après le dernier temps observé non censuré, I'estimation de la survie sera considérée comme non définie. comparées. Dans cet article, nous présentons les résultats de l'application de la méthode non paramétrique à l'exploration de nos données.

Lapproche non paramétrique se rapproche d'une analyse des statistiques descriptives des durées. La fonction de survie est estimée à l'aide de l'estimateur (KM) du produit limite de Kaplan-Meier (1958). Lestimateur KM de la survie à la date $t_{j}$ est calculé comme le produit des proportions suivantes:

$S_{K M}\left(t_{j}\right)=\prod_{k=1}^{j} \frac{n\left(t_{k}\right)-d\left(t_{k}\right)}{n\left(t_{k}\right)}$

où $n\left(t_{k}\right)$ est la population à risque à la date $t_{k}$ et $d\left(t_{k}\right)$ le nombre d'interruptions à la date $t_{k}$. La fonction de survie correspondante est alors une fonction en paliers avec une marche pour chaque temps discret correspondant à une interruption ${ }^{(14)}$. L'estimateur KM considère les probabilités de survie à chaque palier, puis les multiplie pour obtenir un estimateur de la survie ${ }^{(15)}$. 
(16) Les tests d'équivalence des survies entre classes sont basés sur les tableaux de contingence à chaque date, à partir desquels sont testées les différences entre le nombre d'interruptions observées dans une classe et le nombre d'interruptions prédites à partir de l'estimation d'une survie commune à toutes les classes.
Lapplication de cette méthode non paramétrique produit donc un estimateur de la survie pour chaque date. L'étude de cet estimateur et de ses propriétés statistiques permet de construire l'intervalle de confiance de la survie et de tester l'égalité des survies selon différents critères. Particulièrement nous testerons l'équivalence des fonctions de survie des coopératives face aux autres formes juridiques, l'équivalence de ces fonctions pour les coopératives suivant les différents secteurs d'activité et suivant les différentes régions. Cette approche produit une approximation purement empirique des fonctions de hasard et de survie qui est donc a priori proche de la réalité. Mais elle modélise difficilement l'effet des covariables. Dans notre application, seuls des tests ont pu être mis en œuvre pour examiner les relations entre les durées et les covariables ${ }^{(16)}$.

\section{Résultats}

Les résultats obtenus sont présentés en quatre grands points: un premier panorama général des industries agroalimentaires et du commerce, une analyse des coopératives par rapport aux autres entreprises et la mise en évidence des diversités sectorielles, puis régionales de la survie coopérative.

\section{Le panorama général:}

\section{une survie différenciée selon les secteurs d'activité}

Comme le montre la figure 2, la comparaison par grands secteurs d'activité est éclairante des processus de restructuration industrielle à l'œuvre. Les tests mobilisés permettent de rejeter l'hypothèse d'identité des fonctions de survie selon les secteurs $(\mathrm{p}<0,001)$. Le secteur de la viande et

\section{Figure 2}

\section{La survie des entreprises} selon le secteur d'activité économique principal

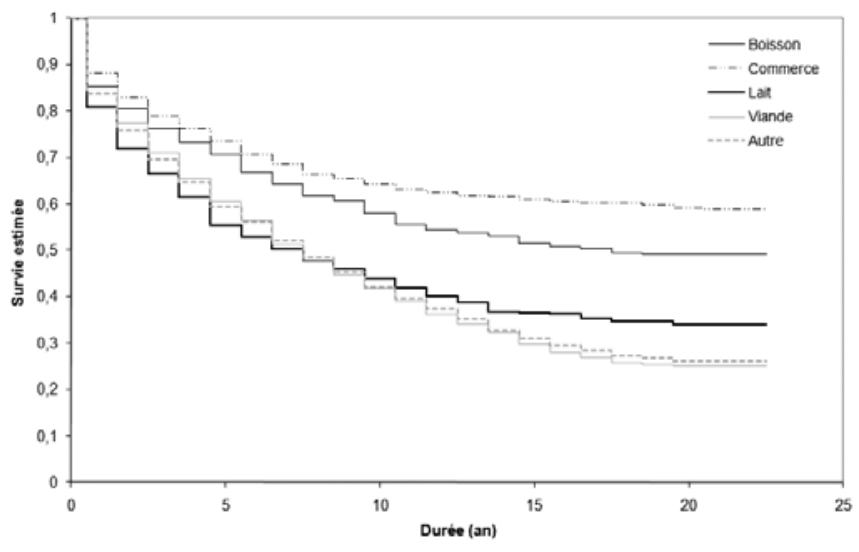


(17) C'est ce que nous montrons par ailleurs dans une autre étude (en cours de parution) avec la mobilisation d'une méthodologie plus complexe car multivariée. le secteur résiduel (" Autre ») comptent les entreprises ayant la plus grande probabilité de disparaître: ainsi, la probabilité de survie est de 0,42 pour les entreprises des deux secteurs au bout de dix ans, et même de 0,25 pour la viande et de 0,26 pour le secteur résiduel au bout de vingt ans. Avec l'évolution législative au cours de la période considérée (et notamment les lois de 1991-1992 ayant modifié le statut coopératif avec la facilitation de la filialisation et l'incitation à la transformation de Sica en coopérative), des effets structurels de période peuvent toutefois être suspectés ${ }^{(17)}$. Comptant une probabilité de survie similaire au bout de dix ans $(0,46)$, le secteur du lait se différencie par une meilleure résistance au-delà de cette période, avec une probabilité de 0,34 au bout de vingt ans. Les entreprises laitières ayant "résisté » à la période forte de restructuration des années 1990-1995 sont donc plus résistantes que celles des secteurs précédents. Les entreprises du secteur de la boisson sont moins sujettes à la disparition: ainsi, il faut attendre dix-huit ans pour que la probabilité de survie passe à 0,5 . Cela suggère que les fortes restructurations de ce secteur, dont a pu parler Touzard (2000), ont d'abord été le fait des petites entreprises viticoles. Enfin, le secteur du commerce de gros de produits alimentaires compte la probabilité de survie la plus importante sur l'ensemble de la période: 0,64 au bout de dix ans et même 0,59 au bout de vingt ans.

\section{Entre coopératives et autres types d'entreprise:}

\section{une plus grande survie des coopératives}

Suivant la figure 3 et les résultats des tests mobilisés ( $\mathrm{p}<0,001)$, la comparaison des fonctions estimées de survie corrobore la première proposition (la plus grande capacité des coopératives à survivre): on constate

\section{Figure 3}

\section{La survie des entreprises selon leur statut juridique}

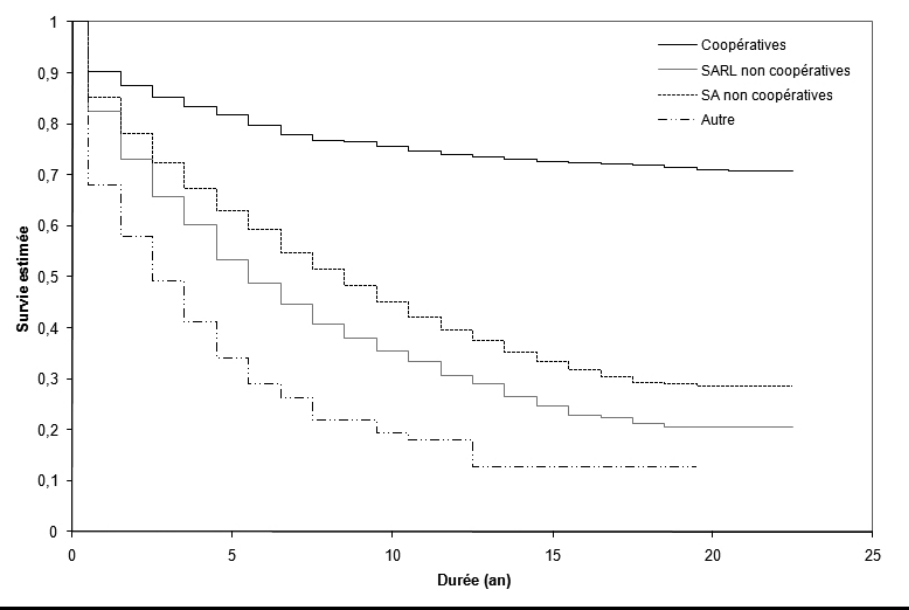


nettement une survie plus grande des coopératives par rapport aux autres entreprises. La probabilité de survie est de 0,76 au bout de dix ans et de 0,7 au bout de vingt ans. On voit en outre que l'essentiel de la diminution de cette probabilité de survie se fait les premières années, la baisse étant plus incrémentale par la suite. Deux structures juridiques ont une dynamique comparable l'une à l'autre: les SA non coopératives et les SARL non coopératives. Il est intéressant de souligner la probabilité de survie des SA non coopératives systématiquement supérieure à celle des SARL non coopératives (respectivement 0,45 et 0,35 au bout de dix ans). A la différence des coopératives, le rythme de baisse de la survie est régulier au cours des années. Enfin, la catégorie résiduelle (regroupant les personnes physiques, les autres personnes morales ou celles de droit public) compte les entreprises ayant la plus faible probabilité de survie. Cette dernière est en effet de 0,19 au bout de dix ans et même de 0,13 au bout de vingt ans.

\section{Une dynamique différenciée des coopératives} au sein des secteurs d'activité

S'intéresser uniquement aux coopératives permet de voir s'il n'y a pas un effet sectoriel. Les tests d'égalité des survies selon les secteurs soulignent au contraire l'existence d'une hétérogénéité forte du système coopératif à ce propos. On constate, comme le montre la figure 4, que la dynamique des coopératives au sein de chaque secteur d'activité n'est pas toujours similaire à la dynamique générale du secteur. Hormis le secteur de la viande, les coopératives agricoles apparaissent comme ayant une forte probabilité de survie. Les coopératives viticoles sont celles qui ont la plus grande chance d'exister quelle que soit la durée considérée $(0,8$ au bout de

\section{Figure 4}

La survie des coopératives selon les secteurs

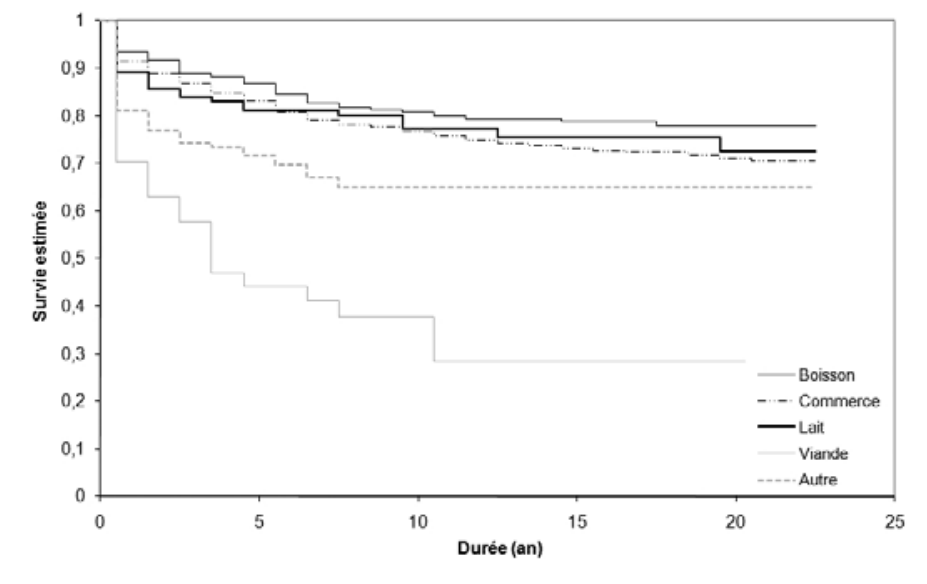


dix ans et 0,78 au bout de vingt ans). Les secteurs du commerce de gros de produits alimentaires et du lait sont assez proches. Les coopératives y font preuve d'une bonne résistance: probabilité de survie de 0,77 au bout de dix ans et de 0,71 (commerce) à 0,72 (lait) au bout de vingt ans. La probabilité de survie des coopératives du secteur de la viande n'est que de 0,38 au bout de dix ans et de 0,28 au bout de vingt ans.

\section{Une dynamique différenciée des coopératives suivant les régions}

L'analyse des survies différenciées par l'appartenance aux régions coopératives illustre une relative hétérogénéité des survies des coopératives relatives aux situations géographiques. La figure 5 et les tests statistiques soutiennent des différences significatives. Nous pouvons clairement identifier, sur le graphique, que les coopératives de la région Nord-Est et celles de la région Centre-Limousin-Poitou sont caractérisées par les meilleures survies de l'échantillon, à chaque date $(0,87$ et 0,8 au bout de dix ans et 0,85 et 0,77 au bout de vingt ans). A l'opposé, la survie la plus faible est associée aux coopératives de Franche-Comté ( 0,56 au bout de dix ans et 0,50 au bout de vingt ans). Au-delà de dix ans, les coopératives des régions Nord-Bassin parisien, Aquitaine et Corse-Languedoc-Paca ont une probabilité de survie proche de 0,70, alors que pour les régions Bourgogne, Midi-Pyrénées, Rhône-AlpesAuvergne et Ouest cette probabilité est proche de 0,76 . Et au-delà de vingt ans, ces probabilités deviennent respectivement 0,65 et 0,70 . Un approfondissement de cette étude consistera à apprécier l'importance des différences sectorielles ou de l'histoire propre à ces régions dans une explication de cette hétérogénéité.

\section{Figure 5 \\ La survie des coopératives selon les régions coopératives}

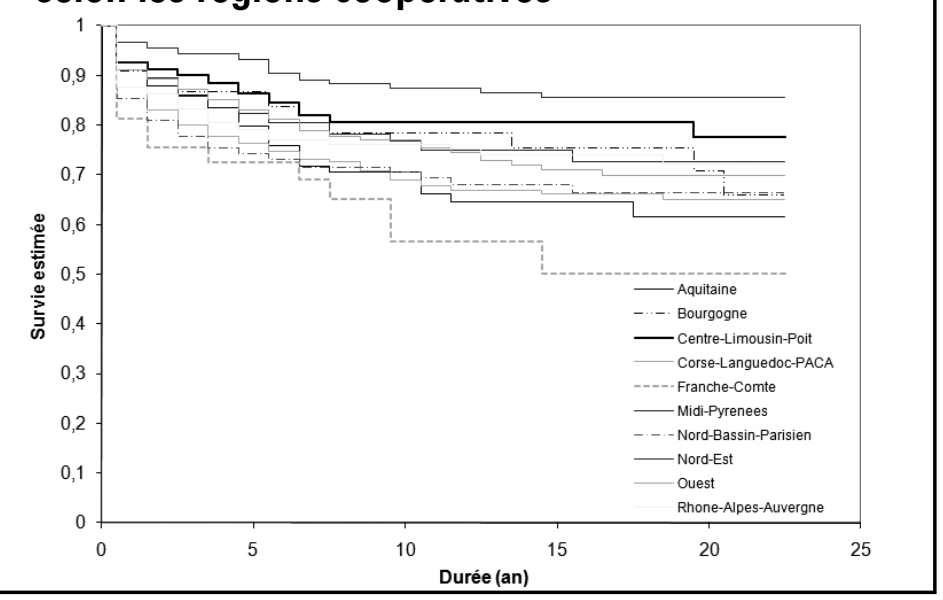




\section{Discussion et conclusion}

Cet article est une première analyse empirique de la survie des coopératives agricoles. Par rapport à la littérature théorique sur la question, nous avons pu mettre en évidence certaines conjectures de travail. Ces conjectures ont été testées à partir d'un modèle simple d'analyse de survie. Nous avons pu ainsi souligner que les coopératives ont une plus grande capacité à survivre que les autres formes d'entreprise.

Une dynamique différenciée selon les secteurs d'activité a pu être mise en évidence.

- Des secteurs industriels (viande) ont été fortement restructurés avec une très faible capacité à survivre des coopératives. Il s'agit de voir dans quelle mesure les coopératives n'ont pas su intégrer la filière de production en raison d'une mobilisation de capitaux importants dans ce secteur. Les évolutions récentes, et notamment le rapprochement d'entreprises coopératives et d'entreprises à capitaux familiaux, semblent aller dans ce sens. Il semble toutefois que ce secteur est de plus en plus investi par des entreprises (coopératives ou non) polyvalentes. Centrée sur la prise en compte des entités ayant pour activité économique principale le secteur de la viande, notre méthodologie doit donc être étendue à une prise en compte de la diversification des activités au sein d'une même organisation.

- Des secteurs ont été fortement restructurés (lait, boisson) avec une forte résistance des coopératives. Les coopératives auraient pu se transformer et trouver des modèles (diversification à base territoriale, filialisation) leur permettant de réagir aux modifications de l'environnement (avec, par exemple, le retrait effectif ou à venir de la Politique agricole commune). La création de filiale commune aux pôles laitiers des grandes coopératives de l'Ouest en est un bon exemple. Cette étude doit toutefois être complétée par une réflexion sur la dynamique propre des petites coopératives (de moins de dix salariés).

- Un secteur résiste en s'appuyant fortement sur les coopératives (commerce). La restructuration de ce secteur s'inscrit sur une plus grande durée et la proposition théorique de voir les coopératives comme permettant des gains d'échelle importants apparaît validée.

La coopérative apparaît comme étant une forme bien adaptée aux transformations actuelles de l'agriculture. Son adaptabilité en raison même de son mode de distribution des pouvoirs la rend plus résistante à une évolution de son environnement. On constate ainsi que l'essentiel de la mortalité des coopératives se déroule les premières années, suggérant donc qu'après une première adaptation une coopérative a tendance à fortement s’ancrer dans son territoire et son secteur. La différence de mortalité soulignée suivant les territoires doit être précisée. On voit alors toute la nécessité pour les fédérations et les organismes de soutien d'intervenir dans les premières années, non seulement en amont, mais également en accompagnement post-création, pour consolider les expériences coopératives. 
(18) Voir les travaux de Jean-Marc Touzard (2000) à ce propos.
Pour les grandes et anciennes coopératives, la filialisation avait été vue comme l'esquisse du développement du passage d'une coopération d'usagers à une coopération d'actionnaires (Forestier et Mauget, 2000). L'évolution du périmètre coopératif avec un nombre croissant de salariés hors de la structure coopérative (Filippi et al., 2008a, 2008b) semble avoir une autre signification dans le cadre de notre étude. Il semble que l'utilisation de la filiale peut être vue non comme un moyen de développer une coopé-rative d'actionnaires, mais comme un moyen d'externaliser le risque de marché, voire de mobiliser des capitaux externes. Un prolongement de cette recherche sera d'explorer plus précisément ce lien entre la filialisation et la survie.

On peut toutefois se demander si dans certains secteurs nous n'assistons pas à l'émergence d'un dualisme fort du système coopératif. En effet, alors qu'Ambiaud (2007) a pu mettre en évidence une disparition continue des petites coopératives, nos résultats montrent une résistance forte des grandes. L'essentiel des disparitions semble donc être le fait de ces petites coopératives. C'est particulièrement le cas dans le secteur viticole et dans celui du commerce de fruits et légumes. Selon l'auteur, ces disparitions semblent renvoyer à deux dynamiques différentes: une logique de concentration continue dans le cadre de la production de vin (plus ouverte à la concurrence internationale) ${ }^{(18)}$; une logique de faillite dans le commerce de gros, où les coopératives disparaissent en raison de la part croissante des entreprises privées de distribution, laissant les agriculteurs soit négocier en direct avec des structures non coopératives, soit adhérer à une coopérative déjà efficace car bénéficiant de fortes économies d'échelle. Cela souligne un élément à approfondir : derrière les disparitions, on trouve en effet des faillites, des entreprises fermées ou des fusions. En complément à une approche paramétrique de la survie des coopératives, une étude des déterminants de la performance économique des entreprises sera menée pour voir en quoi les techniques de production des coopératives se différencient des autres entreprises et en quoi elles sont plus ou moins efficaces. L'étude de l'impact de l'absorption d'une autre entreprise sur la performance de l'entreprise, ce lieu commun des études managériales, conduira donc à compléter cette approche de la survie des coopératives par une analyse de leur efficacité. 


\section{Bibliographie}

Allison P., 1995, Survival analysis using SAS, Cary, SAS Publishing.

André C., Delorme R., 1983, L'Etat et l'économie, Paris, Le Seuil.

Ambiaud E., 2007, "La petite coopération agricole poursuit sa réorganisation ", Agreste primeur, $\mathrm{n}^{\circ} 201$, octobre.

Ambiaud E., 2009, « 2500 coopératives agroalimentaires ", Agreste primeur, n ${ }^{\circ} 220$, janvier. Bager T., 1994, "Isomorphic processes and the transformation of cooperatives ", Annals of public and cooperative economics, $\mathrm{n}^{\circ}$ 65(1), p. 35-57.

Boisard P., Letablier M.-T., 1987, « Le camembert: normand ou normé, deux modèles de production dans l'industrie fromagère ", in Entreprise et Produits, Paris, PUF, "Cahiers du CEE ».

Boyer R., 2005, "Coherence, diversity, and the evolution of capitalism: The institutional complementary hypothesis ", Evolutionary and Institutional Economics Review, $\mathrm{n}^{\circ} 2(1)$, p. 43-80.

Briscoe R., Ward M., 2006, "Is small both beautiful and competitive? A case study of Irish dairy cooperatives ", Journal of rural cooperation, $\mathrm{n}^{\circ}$ 34(2), p. 119-138.

Bureau J.-C., 2007, La Politique agricole commune, Paris, La Découverte, "Repères ".

Côté D. (édit.), 2001, Les holdings coopératifs: évolution ou transformation définitive? Bruxelles, De Boeck Université.

DiMaggio P. J., Powell W., 1983, « The iron cage revisited: Institutional isomorphism and collective rationality in organizational fields ", American Sociological Review, n 48, p. $147-160$.

Draperi J.-F., Touzard J.-M. (édit.), 2003, Les coopératives entre territoires et mondialisation, Paris, L'Harmattan, "Les Cahiers de l'économie sociale».
Filippi M., Frey O., Mauget R., 2008a, « Les coopératives agricoles face à l'internationalisation des marchés", Recma, n 310, p. 31-51. Filippi M., Frey O., Triboulet P., 2008b, "Typologie spatiale des groupes coopératifs agricoles français », in Draperi J.-F. (edit.), Défis coopératifs, Paris, L'Harmattan, "Les Cahiers de l'économie sociale ", p. 17-35.

Forestier M., Mauget R., 2000, "De la coopérative au groupe coopératif agroalimentaire, $1^{\text {re }}$ partie: de la valeur pour l'usager à la valeur pour l'actionnaire? ", Recma, n 278. Gagliardi F., 2009, « Financial development and the growth of cooperative firms ", Small Business Economics, $\mathrm{n}^{\circ}$ 32, p. 39-464.

Hansmann H., 1996, The ownership of enterprise, Cambridge, Harvard University Press. Hansmann H., 1999, "Cooperative firms in theory and practice ", Finnish Journal of business economics, 48(4), p. 387-403.

Harte L., O’Connell J., 2007, «European dairy cooperative strategies: Horizontal integration versus diversity ", in Karantininis K., Nilsson J. (eds.), Verticalmarkets and cooperative hierarchies, Dordrecht, Springer, p. 195-211. Heckman J., 2008, "Econometric causality ", International Statistical Review, n ${ }^{\circ}$ 76(1), p. 1-27.

Hosmer D., Lemeshow S., 1999, Applied survival analysis, New York, Wiley \& Sons.

Jarrigue F., Touzard J.-M., 2001, «Les mutations de l'organisation coopérative à travers l'évolution de ses règles ", Recma, $\mathrm{n}^{\circ} 280$, avril.

Kalbfleisch J. D., Prentice R. L., 1980, The statistical analysis offailure time data, New York, Wiley \& Sons.

Kaplan E., Meier P., 1958, « Non-parametric estimation from incomplete observations", Journal of the American Statistical Association, $\mathrm{n}^{\circ} 53$ (282), p. 457-481. 
Koulytchizky S., Mauget R., 2003, "Le développement des groupes coopératifs agricoles depuis un demi-siècle ", Recma, n 287, p. 14-40.

Lawless J.-F., 2003, Statistical models and methods for lifetime data, New York, Wiley $\&$ Sons.

Maietta O. W., Sena V., 2008, «Is competition really bad news for cooperatives?", Journal of productivity analysis, $\mathrm{n}^{\circ} 29$, p. 221-233.

Moati P., Loire S., Medan P., 1997, Démographie des entreprises: une revue de la littérature, Paris, Cahiers de recherche du Credoc.

Mosheim R., 2008, « Efficiency and survival: the impact of the international coffee agreement's demise on Costa Rica's cooperative coffee processing 1988-2005 ", Annals of public and cooperative economics, $\mathrm{n}^{\circ}$ 79(1), p. 79-106. Mucchielli J.-L., Kohler P., 2000, "Déterminants et conséquences des fusionsacquisitions ", Revue française de gestion, $\mathrm{n}^{\circ} 131$, novembre-décembre, p. 6-19.

Réquillard V., 2007, "Coûts de production, gains de productivité et prix des fruits et légumes ", in Combris P., Amiot-Carlin M.-J., Caillavet F., Causse M., Dallongeville J., Padilla M., Renard C., Soler L.-G. (édit.), Les fruits et légumes dans l'alimentation: enjeux et déterminants de la consommation, Paris, Inra, unité expertise scientifique, p. 306-320.

Rey P., Tirole J., 2000, Régulation des relations entre fournisseurs et distributeurs, rapport $d u$ Conseil d'analyse économique, Paris, La Documentation française.
Rey P., Tirole J., 2007, « Financing and access in cooperatives ", International Journal of industrialorganization, $\mathrm{n}^{\circ} 25(5)$, p. 1061-1088. Rousselière D., 2009, "What is economic democracy? An inquiry into French cooperatives ", Studies in political economy, no 84, p. 29-46.

Rousselière D., Vézina M., 2009, "Constructing the legitimacy of a financial cooperative in the cultural sector: A Case study using textual analysis ", International Review of sociology, n $n^{\circ} 19(2)$, p. 241-261.

Simons T., Ingram P., 2004, «An ecology of ideology: Theory and evidence from four populations ", Industrial and Corporate Change, no 13(1), p. 33-59.

Singh S., Coelli T., Fleming E., 2001, "Performance of dairy plants in the cooperative and private sectors in India ", Annals of public and cooperative economics, $\mathrm{n}^{\circ} 72(4)$, p. 453-479.

Spear R., 2000, "The co-operative advantage ", Annals of public and cooperative economics, $\mathrm{n}^{\circ} 71(4)$, p. 507-523.

Sykuta M., Cook M.-L., 2001, « A New institutional economics approach to contracts and cooperatives ", American Journal of agricultural economics, $\mathrm{n}^{\circ}$ 83(5), p. 1273-1279.

Touzard J.-M., 2000, « Coordinations locales, innovation et régulation: l'exemple de la transition "vin de masse"-"vins de qualité" en Languedoc-Roussillon", Revue d'économie régionale et urbaine, $\mathrm{n}^{\circ} 3-00$, p. 589-604.

Vienney C., 1994, L'économie sociale, Paris, La Découverte, «Repères ». 